\title{
Disability Status and Its Influencing Factors Among Stroke Patients in Northeast China: A 3-Year Follow-Up Study
}

\author{
Yumei Lv' \\ Qiuxue Sun' \\ Juan $\mathrm{Li}^{2}$ \\ Wenyue Zhang ${ }^{3}$ \\ Yudi $\mathrm{He}^{\prime}$ \\ Yuqiu Zhou' \\ 'School of Nursing, Harbin Medical \\ University (Daqing), Daqing, Heilongjiang \\ Province, People's Republic of China; \\ ${ }^{2}$ Department of Pneumology, Qingdao \\ Hospital of Traditional Chinese Medicine, \\ Qingdao, Shangdong Province, People's \\ Republic of China; ${ }^{3}$ Department of \\ Rehabilitation, People's Hospital of \\ Daqing, Daqing, Heilongjiang Province, \\ People's Republic of China
}

Correspondence: Yuqiu Zhou

School of Nursing, Harbin Medical

University (Daqing), No. 39 Xinyang

Road, Longfeng District, Daqing, 163319 ,

Heilongjiang Province, People's Republic

of China

Email hlxzyq@126.com
Purpose: To explore the rate of post-stroke disability and its associated factors in the third year following discharge from inpatient rehabilitation in Northeast China.

Design: A prospective cohort study.

Methods: A total of 522 persons who were hospitalized with a diagnosis of stroke were recruited consecutively between April 2015 and December 2015 and followed for 3 years. The primary outcome was disability, which was assessed using the Modified Barthel Index (MBI), a cutoff score of $\leq 95$ indicates disability. Plausible risk factors of disability were selected from available variables to perform multivariate logistic regression analysis.

Findings: The proportion of post-stroke patients with disability decreased from $63.8 \%$ to $46.7 \%$ at 3-year follow-up. The factors associated with post-stroke disability were age, neurological deficits, cognitive function, depression, and social support.

Conclusions/Clinical Relevance: Disability continues to be a significant issue for individuals after a stroke, and community health workers should perform targeted assessments and interventions to decrease disability, and pay special attention to individuals who are at greatest risk of post-stroke disability.

Keywords: stroke, disability, influencing factors

\section{Background}

Stroke is the leading cause of death globally, particularly in China, while survival may be accompanied by severe chronic disabilities. ${ }^{1}$ Permanent sequelae causing problems with everyday activities and functions may have negative effects on patients' self-care ability and social participation, significantly reduce the healthrelated quality of life.,3 Growing evidence highlights that timely treatment and early rehabilitation is beneficial in improving patient outcomes, such as functional status, length of stay and discharge destination. ${ }^{4,5}$ Few studies have reported the long-term recovery of stroke patients discharged from inpatient rehabilitation, and the results are inconsistent, therefore, it is unclear whether those patients are able to sustain their recovery progress from early rehabilitation.

As stroke is more prevalent in Northeast China, ${ }^{6}$ the resulting healthcare burden of long-term post-stroke disability in this region is increased, which may be affected by various influential factors (such as level of regional medical services, awareness of rehabilitation and environmental factors). Thus the identification and prevention of long-term post-stroke disability in Northeast China and its influencing factors have assumed major importance. Nowadays the reported disability rates in 
Northeast China vary widely between different studies, most of which conduct cross-regional research that focuses on a single factor's influence on disability and short-term data. ${ }^{7,8}$ Longitudinal studies of long-term post-stroke disability are few in number. Sustainable interventions to strengthen long-term recovery need to be investigated. Accordingly, we conducted a survey in Northeast China, and aimed to explore the disability rate and associated factors among disabled patients during the third year following stroke. We hope that the findings of our study will provide a reference for the implementation of targeted interventions, help community health workers raise their awareness of further research/practice in stroke patients with long-term disability.

\section{Materials and Methods}

\section{Design}

This cohort study of stroke patients forms one part of a project supported by the National Natural Science Foundation of China (\#71303072). A total of 689 patients from 4 hospitals in Daqing were consecutively recruited via stratified random sampling to take part in the project (ie, the risk factors of post-stroke disability within a structural equation model) between April 2015 and December 2015. The sample size estimation was calculated by using the sample size calculation formula. Patients were recruited within one month of the onset of stroke. The current study has a longitudinal design of which focused on physical activity long-term after stroke. Patients who are able to completed the follow-up assessments after discharge to their home were potential participants. Among the original sample, 522 participants met the inclusion criteria. Inclusion criteria were: 1) age $\geq 18$ years; 2) patients who were hospitalized in the department of rehabilitation with a diagnosis of stroke by computed tomography or magnetic resonance imaging; 3) patients who completed the clinical interview by telephone call after discharge to their home; 4) patients who provided informed consent. Exclusion criteria were: patients with severe cognitive impairment (MMSE $<10$ ), global aphasia, other major physical or psychiatric conditions, and history of drug dependence. At the time of the follow-up assessment, 23 participants died and 51 were lost to follow-up. A total of 448 participants was therefore used, met the requirement for a logistic regression analysis. The flowchart of the study population is illustrated in Figure 1. The study protocol was approved by the Ethics Committee of

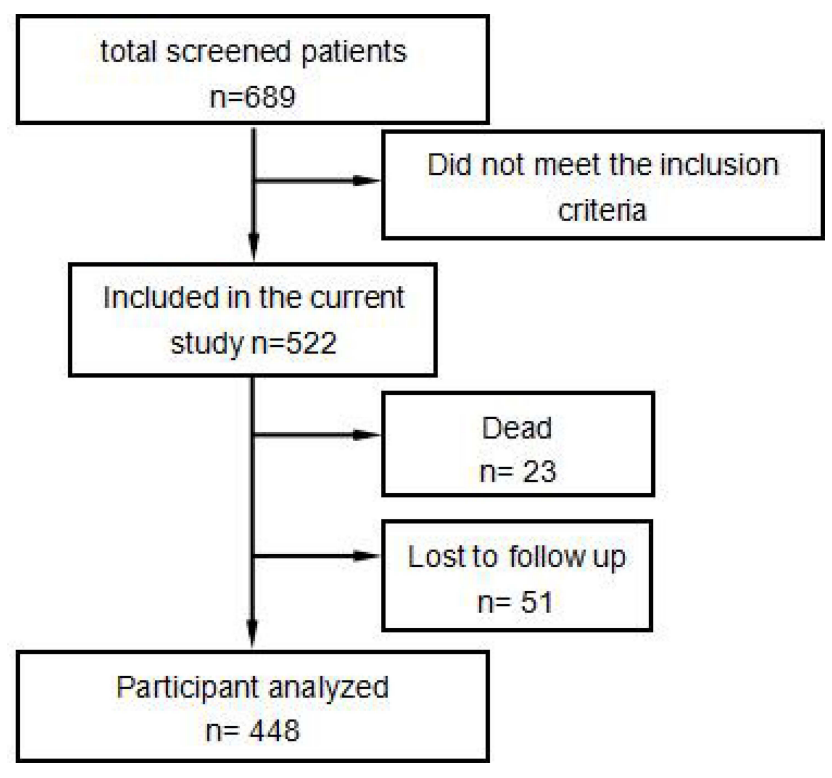

Figure I Flowchart of the study population.

the Harbin Medical University (Daqing Branch) and the participating hospitals (No 2015-03). Written informed consent was obtained from each participant prior to inclusion in the study. This study was conducted in accordance with the Declaration of Helsinki.

\section{Data Collection}

At baseline, sociodemographic (age, gender, occupation, marital status, educational level, and income status), clinical (stroke type and frequency, lesion location, course of disease, extent of neurological functional deficit, medical history, comorbidities, and impairments), and psychosocial data were collected by face-to-face administration of questionnaires during interviews in the hospital setting when they were discharged. The follow-up assessments including stroke mortality and physical disability were conducted via telephone interview. All interviews were conducted by the primary researchers who had been trained in a use of standardized procedures.

The extent of neurological functional deficit was assessed by the National Institutes of Health Stroke Scale (NIHSS) over a range of 0-42 points, a lower score being better. ${ }^{9}$ The Social Support Rating Scale (SSRS, 0-42 points, higher score is better) was used to measure the patients' social support level. The SSRS includes subscores for objective support, subjective support, and support utilization. ${ }^{10}$ The Self-rating Depression Scale (SDS) was used to assess severity of depression. ${ }^{11}$ Cognition function was measured by the Mini Mental State 
Examination (MMSE). Cognitive impairment was defined as an MMSE total score of $<24$. $^{12}$

Post-stroke disability status was assessed using the modified Barthel Index (mBI), with a score less than 95 meeting the criteria for post-stroke disability. ${ }^{13,14}$ The mBI is a 10-item instrument that measures a person's degree of independence in daily life. The total scores can range from 0 to 100 , with lower scores representing less independent functioning ( 0 represents total assistance and 100 complete independence). This instrument has previously been found to be a reliable measure of functional independence for survivors of stroke (Cronbach's alpha $=0.93$ ), with interrater correlations ranging from 0.86 to 0.88 .

\section{Analysis}

The data were processed and analyzed using IBM SPSS 20.0. The level of significance for all analyses was set as $P<0.05$. Dichotomous variables (age, gender, occupation, marital status, educational level, stroke type, previous stroke, monthly family income, hypertension, and diabetes) were compared by chi-square test. Continuous variables (NIHSS, MMSE, SDS, GSES, and SSRS scores) were compared by independent sample $t$-test or Wilcoxon test. Multivariate logistic regression analysis was used to identify the independent influencing factors of post-stroke disability.

\section{Results}

\section{Profile of Study Sample}

A total of 522 patients who fulfilled the inclusion and exclusion criteria were included in the study, after excluding 74 cases, 448 patients were evaluated. Among the 448 patients, 281 were male $(62.7 \%) ; 88.8 \%$ were married, $83.3 \%$ suffered ischemic stroke, $28.3 \%$ had diabetes, $73.9 \%$ had hypertension, $19 \%$ had heart disease, and $40.4 \%$ had previous stroke. Most patients were manual workers (32.1\%) and non-manual workers (29.2\%).

\section{Disability}

At baseline, 333 patients met the criteria for disability. The rate of disability was $63.8 \%$ and the proportion of mild, moderate, severe, and totally disability was 30.1\% (157/ 522), 18.0\% (94/522), 10.7\% (56/522), and 5.0\% (26/522), respectively. During the 3-year follow-up, 209 (46.7\%) patients met the criteria for disability. The proportion of mild, moderate, severe, and total disability was $25.9 \%$ (116/ 448), 7.4\% (33/448), 8.3\% (37/448), and 5.1\% (23/448), respectively. Compared with the group without disability, those with disability in the third year of follow-up were more likely to be older and solitary, with intracerebral hemorrhage, previous history of stroke, higher NIHSS and SDS scores, and lower MMSE, GSES, and SSRS scores (Table 1).

\section{Factors Associated with Disability}

As shown in Table 1, the $P$ values for age, gender, marital status, stroke type, previous stroke, hypertension, NIHSS score, MMSE score, SDS score, GSES score, and SSRS score were less than 0.2 , and we included these factors as candidates in a multivariate logistic regression model to examine their independent association with 3-year disability. Logistic regression showed that the independent influencing factors for post-stroke disability were age, neurological deficits, cognition function, depression, and social support (Table 2).

\section{Discussion}

Our study has a longitudinal design of which focused on long-term disability after stroke. This method was selected as it is especially suitable to describe the change in disability. To our knowledge, this is a rare study to explore the rate of post-stroke disability and its associated factors in the third year following discharge from inpatient rehabilitation in Northeast China. We present a multidisciplinary perspective about rehabilitation potential and goals. In this 3-year-long follow-up study, patients showed improvement in overall function after discharge from inpatient rehabilitation. The proportion of post-stroke patients with disability decreased from $63.8 \%$ to $46.7 \%$. The rate of disability in the third year was $42.6 \%$ in ischemic stroke patients and $65.3 \%$ in hemorrhagic stroke patients. Although the disability rate decreased substantially, it is still higher than that reported in most studies. $^{15,16}$ For example, a Brazilian study reported a disability rate of $18 \%$ in the third year after stroke, while a longitudinal study of functional outcome after stroke conducted in Sweden reported 40.9\%. ${ }^{17,18}$ The wide variation in study results may be due to differences in the assessment of disability and study setting. In the current study post-stroke disability was assessed by $\mathrm{mBI}$, which demonstrated satisfactory factorial validity and internal consistency, and reflected subtle changes in functional outcome. This may have resulted in a relatively high rate of post-stroke disability. Here we considered 95 as the cut-off value of post-stroke disability, compared with 85 in a Brazilian study and 100 in a Singaporean study. ${ }^{19,20}$ 
Table I Comparison of Factors Between Stroke Patients with and without Disability in the Third Year of Follow-Up

\begin{tabular}{|c|c|c|c|c|c|c|}
\hline \multirow[t]{2}{*}{ Factors } & \multicolumn{2}{|c|}{ Disability n= 209} & \multicolumn{2}{|c|}{ No Disability $n=239$} & \multirow[t]{2}{*}{$x^{2}$} & \multirow[t]{2}{*}{$\mathbf{P}$} \\
\hline & $\mathbf{n}$ & $\%$ & $\mathbf{n}$ & $\%$ & & \\
\hline \multicolumn{7}{|l|}{ Age } \\
\hline$<50$ & 17 & 8.1 & 29 & 12.1 & 18.818 & 0.001 \\
\hline $50 \sim 59$ & 38 & 18.2 & 64 & 26.8 & & \\
\hline $60 \sim 69$ & 68 & 32.5 & 92 & 38.5 & & \\
\hline $70 \sim 79$ & 67 & 32.1 & 41 & 17.2 & & \\
\hline$\geq 80$ & 19 & 9.1 & 13 & 5.4 & & \\
\hline \multicolumn{7}{|l|}{ Gender } \\
\hline Male & 124 & 59.3 & 157 & 65.7 & 1.929 & 0.172 \\
\hline Female & 85 & 40.7 & 82 & 34.3 & & \\
\hline \multicolumn{7}{|l|}{ Marital status } \\
\hline Married & 176 & 84.2 & 222 & 93.9 & 8.466 & 0.004 \\
\hline Unmarried/divorced/widowed & 33 & 15.8 & 17 & 6.1 & & \\
\hline \multicolumn{7}{|l|}{ Month family earning } \\
\hline$<5000$ & 109 & 52.2 & 111 & 46.4 & 1.454 & 0.256 \\
\hline$\geq 5000$ & 100 & 47.8 & 128 & 53.6 & & \\
\hline \multicolumn{7}{|l|}{ Stroke type } \\
\hline Ischemic stroke & 159 & 76.1 & 214 & 89.5 & 15.894 & 0.000 \\
\hline Intracerebral hemorrhage & 47 & 22.5 & 25 & 10.5 & & \\
\hline Mixed & 3 & 1.4 & 0 & 0 & & \\
\hline \multicolumn{7}{|l|}{ Previous stroke } \\
\hline Yes & 95 & 45.5 & 86 & 36.0 & 4.154 & 0.043 \\
\hline No & 114 & 54.5 & 153 & 64.0 & & \\
\hline Hypertension & 161 & 77.0 & 170 & 71.1 & 2.014 & 0.163 \\
\hline \multirow[t]{2}{*}{ Diabetes } & 60 & 28.7 & 67 & 28.0 & 0.025 & 0.916 \\
\hline & $\overline{\mathbf{x}}$ & SD & $\overline{\mathbf{x}}$ & SD & $t$ & $\mathbf{P}$ \\
\hline NIHSS & 7.44 & 5.61 & 3.51 & 4.34 & -8.208 & 0.000 \\
\hline MMSE & 21.01 & 7.52 & 26.19 & 4.50 & 8.948 & 0.000 \\
\hline SDS & 43.98 & 10.30 & 35.89 & 8.97 & -8.806 & 0.000 \\
\hline GSES & 21.47 & 10.15 & 28.44 & 9.07 & 7.619 & 0.000 \\
\hline SSRS & 38.06 & 7.22 & 42.09 & 6.38 & 5.334 & 0.000 \\
\hline
\end{tabular}

Different definitions of disability may lead to different conclusions, in addition to geographical differences in research locations. Our study was conducted in Northeast

Table 2 Multiple Regression Analysis of Factors Affecting PostStroke Disability in the Third Year of Follow-Up

\begin{tabular}{|l|l|l|l|}
\hline Factors & $\mathbf{P}$ & OR & $\mathbf{9 5 \%} \mathbf{~ C l}$ \\
\hline Age & 0.038 & 1.263 & $1.013 \sim 1.574$ \\
NIHSS & 0.000 & 1.141 & $1.082 \sim 1.203$ \\
MMSE & 0.003 & 0.936 & $0.896 \sim 0.978$ \\
SDS & 0.014 & 1.033 & $1.007 \sim 1.06 \mathrm{I}$ \\
SSRS & 0.034 & 0.962 & $0.928 \sim 0.997$ \\
\hline
\end{tabular}

China, an area of high prevalence for stroke and hypertension. ${ }^{21}$ Moreover, differences in the level of medical treatment, input of health resources, economic status of residents, and available rehabilitation resources in different geographical regions could also contribute to variations in study results.

Age has been shown to be an independent influencing factor for post-stroke disability in both the present and previous studies, ${ }^{22,23}$ older age being associated with worsening functional outcome. The decreasing ability of the body to compensate with increasing age may result in slower recovery of somatic dysfunction in older patients. 
At the same time, the will and initiative to undergo rehabilitation therapy might also differ between younger and older patients. With the aging population becoming an increasing concern, the problem of rehabilitation care for the aged is gradually assuming greater prominence. Therefore, it is an important task for the social and health service departments to direct more attention to the rehabilitation of older stroke patients to help reduce the disability rate and improve their quality of life.

In this study, there was a significantly higher NIHSS score in the group of patients with disability in comparison with those without disability. In other words, neurological deficit was a major contributor to post-stroke disability, the result is consistent with the findings of previous studies. $^{24,25}$ In this study, we also found that cognitive function was strongly associated with post-stroke disability in the third year of follow-up, the result is consistent with the conclusion drawn by Huang et al. ${ }^{26}$ Cognitive impairment stroke patients may have attention deficit, leading to a decline in understanding and social adaptability, and resulting in negative outcomes in neural functional recovery.

Our findings support the hypothesis that depression had a negative effect on functional outcome, similar to those of previous studies. ${ }^{27,28}$ The occurrence of post-stroke depression was related to the daily activities of stroke patients, the sudden physical symptoms had a negative effect on activities of daily living, and patients developed significant psychological problems arising from these experiences, such as depression, pessimism, and anxiety. ${ }^{29}$ Conversely, these negative emotions were also capable of affecting the recovery of neurological function, and eventually formed a vicious circle. Hence, clinicians should prioritize early detection and early treatment of psychological problems to reduce the occurrence of poststroke depression and improve the overall prognosis of stroke patients. In addition, this study and preliminary evidence have shown that social support plays an important role in the prognosis of stroke patients. ${ }^{30}$ For this reason, the system of social support must be constructed to promote better recovery of these patients.

Decrease disability requires multidisciplinary intervention. It is recommended that all patients with stroke receive a comprehensive multidisciplinary assessment to determine their rehabilitation needs when they return to communities or home. Community health workers play an essential role in promoting rehabilitation and facilitating care transitions for post-stroke patients with disability. ${ }^{31} \mathrm{In}$ order to help patients minimize post-stroke disability and maintain emotional and social aspects of recovery, interventions after discharge implemented by the multidisciplinary team including community health workers are imperative. Disability is a common complication of stroke patients, which is influenced by multiple factors. The findings of this study will help community health workers design and develop personalized intervention strategies for post-stroke patients with disability, addressing specific needs of stroke patients who have characteristics associated with disability, thereby facilitating recovery.

\section{Study Limitations}

The study has limitations. First, as we mainly focused on post-stroke disability in the third year following discharge from inpatient rehabilitation in Northeast China, other outcome variables were not considered in this study. The depth of information gathered was insufficient. For example information on the length of inpatient stay was not recorded. Furthermore, stroke is increasingly recognized as an important cause of cognitive problems and has been implicated in the development of both Alzheimer's disease and vascular dementia. ${ }^{32}$ We did not look at the relationship between them in this study. Second, the multivariate analysis did not take abnormality on the imaging metric into consideration, which could be helpful to analyze neurological deterioration. However, as detailed information about imaging was unavailable, we were unable to perform such analysis. Third, some variables, such as treatments and therapies administered during hospitalization were not included in the present study. Lastly, the duration of follow-up was relatively short.

\section{Conclusion}

The proportion of post-stroke patients with disability decreased from $63.8 \%$ to $46.7 \%$ in the third year of followup. Although there has been improvement in functional independence after stroke, but long-term post-stroke disability remains a cause for concern. The independent influencing factors for post-stroke disability were age, neurological deficits, cognition function, depression, and social support. Considering the high risk for long-term post-stroke disability, regular screening of stroke patients is warranted. An important task for the social and health service departments in Northeast China will be to focus more attention on the rehabilitation of stroke patients in order to reduce the occurrence of post-stroke depression and improve their prognosis. Particular attention should be directed toward establishing an adequate system of social support, while community health 
workers should adopt a crucial role in providing postdischarge care for stroke survivors and their families.

\section{Disclosure}

The authors report no conflicts of interest in this work.

\section{References}

1. Krishnamurthi RV, Ikeda T, Feigin VL. Global, regional and country-specific burden of ischaemic stroke, intracerebral haemorrhage and subarachnoid haemorrhage: a systematic analysis of the Global Burden of Disease Study 2017. Neuroepidemiology. 2020;54 (2):171-179. doi:10.1159/000506396

2. Tsalta-Mladenov M, Andonova S. Health-related quality of life after ischemic stroke: impact of sociodemographic and clinical factors. Neurol Res. 2021;11:1-9.

3. Zhou X, Du M, Weng Y, et al. Hard return: the development and transformation process of social participation in stroke survivors; a qualitative study and initial theory. Clin Rehabil. 2020;34 (6):824-836. doi:10.1177/0269215520917191

4. Lin RC, Chiang SL, Heitkemper MM, et al. Effectiveness of early rehabilitation combined with virtual reality training on muscle strength, mood state, and functional status in patients with acute stroke: a randomized controlled trial. Worldviews Evid Based Nurs. 2020;17(2):158-167. doi:10.1111/wvn. 12429

5. Purvis T, Kilkenny MF, Middleton S, et al. Influence of stroke coordinators on delivery of acute stroke care and hospital outcomes: an Observational Study. Int $J$ Stroke. 2018;13(6):585-591. doi: $10.1177 / 1747493017741382$

6. Wang W, Jiang B, Sun H, et al. Prevalence, incidence, and mortality of stroke in china clinical perspective: results from a nationwide population-based survey of 480687 adults. Circulation. 2017;135 (8):759-771. doi:10.1161/CIRCULATIONAHA.116.025250

7. Xing L, Jing L, Tian Y, et al. Epidemiology of stroke in urban northeast China: a population-based study 2018-2019. Int J Stroke. 2021;16(1):73-82.

8. Tang SC, Yeh SJ, Tsai LK, et al. Association between plasma levels of hyaluronic acid and functional outcome in acute stroke patients. J Neuroinflammation. 2014;11(10):1-7. doi:10.1186/17422094-11-101

9. Lyden P, Brott T, Tilley B, et al. Improved reliability of the NIH stroke scale using video training. NINDS TPA Stroke Study Group. Stroke. 1994;25(11):2220-2226. doi:10.1161/01.STR.25.11.2220

10. Tang VW, Leung SK, Linda LC. Clinical correlates of the caregiving experience for Chinese caregivers of patients with schizophrenia. Soc Psychiatry Psychiatr Epidemiol. 2008;43(9):720. doi:10.1007/ s00127-008-0357-6

11. Campbell MH, Maynard D, Roberti JW, et al. A comparison of the psychometric strengths of the public-domain zung self-rating depression scale with the proprietary beck depression inventory-II in Barbados. West Indian Med J. 2012;61(5):483-488. doi:10.7727/ wimj.2010.145

12. Liman TG, Heuschmann PU, Endres M, et al. Impact of low minimental status on health outcome up to 5 years after stroke: the Erlangen stroke project. $J$ Neurol. 2012;259(6):1125-1130. doi:10.1007/s00415-011-6312-6

13. Yang H, Chen Y, Wang J, et al. Activities of daily living measurement after ischemic stroke: Rasch analysis of the modified Barthel Index. Medicine. 2021;100(9):e24926. doi:10.1097/MD.00000 00000024926

14. Yang CM, Wang YC, Lee CH, Chn MH, Hsieh CL. A comparison of test-retest reliability and random measurement error of the Barthel Index and modified Barthel Index in patients with chronic stroke. Disabil Rehabil. 2020:1-5. doi:10.1080/09638288.2020.1814429
15. Ganesh A, Luengo-Fernandez R, Wharton RM, et al. Time Course of Evolution of Disability and Cause-Specific Mortality After Ischemic Stroke: implications for Trial Design. J Am Heart Assoc. 2017;6(6): e005788. doi:10.1161/JAHA.117.005788

16. Wang R, Zhang T, Langhammer B. Activities of daily living and life satisfaction of persons with stroke after rehabilitation in China: a Longitudinal Descriptive Study. Top Stroke Rehabil. 2019;26 (2):113-121. doi:10.1080/10749357.2018.1550615

17. Cabral NL, Nagel V, Conforto AB, et al. Five-year survival, disability, and recurrence after first-ever stroke in a middle-income country: a Population-Based Study in Joinvile, Brazil. Int J Stroke. 2018;13 (7):725-733. doi:10.1177/1747493018763906

18. Sennfält S, Norrving B, Petersson J, et al. Long-term survival and function after stroke: a longitudinal observational study from the swedish stroke register. Stroke. 2019;50(1):53-61. doi:10.1161/ STROKEAHA.118.022913

19. Poletto SR, Rebello LC, Valenca MJ, et al. Early mobilization in ischemic stroke: apilot randomized trial of safety and feasibility in a public hospital in Brazil. Cerebrovasc Dis Extra. 2015;5(1):31-40. doi: $10.1159 / 000381417$

20. Kong KH, Lee J. Temporal recovery of activities of daily living in the first year after ischemic stroke: a prospective study of patients admitted to a rehabilitation unit. NeuroRehabilitation. 2014;35(2):221-226.

21. Zhang FL, Liu HY, Wu YH, et al. Hypertension prevalence, awareness, treatment, and control in northeast China: a population-based cross-sectional survey. $J$ Hum Hypertens. 2017;32(1):54-65. doi:10.1038/s41371-017-0003-4

22. Melo LP, Oliveira DC, Dantas A, et al. Predictive factors of functional independence in basic activities of daily living during hospitalization and after discharge of stroke patients. Brain Inj. 2020;35(1):1-6.

23. Oyewole OO, Ogunlana MO, Oritogun KS, et al. Post-stroke disability and its predictors among Nigerian stroke survivors. Disabil Health J. 2016;9(4):616-623. doi:10.1016/j.dhjo.2016.05.011

24. Einstad MS, Saltvedt I, Lydersen S, et al. Associations between post-stroke motor and cognitive function: a cross-sectional study. BMC Geriatr. 2021;21(1):103. doi:10.1186/s12877-021-02055-7

25. Zhao S, Zhang T, Li B, et al. What determines good rehabilitation recovery in stroke patients with very severe disability? A Retrospective Cohort Study. J Stroke Cerebrovasc Dis. 2020;29 (10):105130. doi:10.1016/j.jstrokecerebrovasdis.2020.105130

26. Huang Y, Yang S, Jia J. Factors related to long-term post-stroke cognitiveimpairment in young adult ischemic stroke. Med Sci Monit. 2015;21:654-660. doi:10.12659/MSM.892554

27. López-Espuela F, Roncero-Martín R, Canal-Macías ML, et al. Depressed mood after stroke: predictive factors at six months follow-up. Int J Environ Res Public Health. 2020;17(24):9542. doi:10.3390/ijerph17249542

28. Astuti P, Kusnanto K, Dwi Novitasari F. Depression and functional disability in stroke patients. J Public Health Res. 2020;9(2):1835.

29. Stolwyk RJ, Low T, Gooden JR, et al. A longitudinal examination of the frequency and correlates of self-reported neurobehavioural disability following stroke. Disabil Rehabil. 2020;4:1-9. doi:10.1080/ 09638288.2020.1840637

30. Guo Y, Zhang Z, Lin B, et al. The unmet needs of community-dwelling stroke survivors: a systematic review of qualitative studies. Int J Environ Res Public Health. 2021;18(4):2140. doi:10.3390/ijerph18042140

31. Deng A, Yang S, Xiong R. Effects of an integrated transitional care program for stroke survivors living in a rural community: a randomized controlled trial. Clin Rehabil. 2020;34(4):524-532. doi:10.1177/0269215520905041

32. Slevin M, Matou S, Zeinolabediny Y, et al. Monomeric C-reactive protein-a key molecule driving development of Alzheimer's disease associated with brain ischaemia? Sci Rep. 2015;5(1):1-21. doi:10.1038/srep13281 


\section{Publish your work in this journal}

Neuropsychiatric Disease and Treatment is an international, peerreviewed journal of clinical therapeutics and pharmacology focusing on concise rapid reporting of clinical or pre-clinical studies on a range of neuropsychiatric and neurological disorders. This journal is indexed on PubMed Central, the 'PsycINFO' database and CAS, and is the official journal of The International Neuropsychiatric Association (INA). The manuscript management system is completely online and includes a very quick and fair peer-review system, which is all easy to use. Visit http://www.dovepress.com/testimonials.php to read real quotes from published authors.

Submit your manuscript here: https://www.dovepress.com/neuropsychiatric-disease-and-treatment-journal 